\title{
A Method for Automatic Scaling of Ionograms and Electron Density Reconstruction
}

\author{
Gokhan GOK, Yasar Kemal ALP, Orhan ARIKAN, Member, IEEE, Feza ARIKAN, Member, IEEE
}

\begin{abstract}
Ionogram scaling is the process of reconstructing electron density with respect to height by using the measurements of a remote sensing instrument known as ionosonde. In this study, a novel two stage ionogram scaling technique, ISED, is proposed. In the first stage, Hidden Markov Models (HMMs) are used to identify the actual ionospheric reflections in the ionosonde measurements. In the second stage, an IRI-Plas model based optimization problem is solved to obtain the vertical profile that generates the best least squares fit to the reflections identified in the first stage. To show the performance of ISED in global scale, experiments are conducted on 14,812 ionograms recorded at the three different stations which are Pruhonice in Czech Republic, Eielson in USA and Sao Luis in Brazil. Application of ISED to raw ionograms indicate $97.6 \%$ of the cases, ISED provides accurate electron density reconstructions, which is an improvement about $8.7 \%$ over ARTIST, most commonly used ionogram scaling technique.
\end{abstract}

Index Terms-Ionogram scaling, true height analysis, ARTIST, NhPC, HMM, ionosonde, ISED, Hidden Markov Models.

\section{INTRODUCTION}

Ionosondes are important remote sensing instruments that provide valuable information about the electron density distribution in the ionosphere [1]. They transmit modulated High Frequency (HF) pulses and record their returns from the ionosphere. The set of data that contains round trip delays of the received echoes at different frequencies is called the ionogram, and it provides information about the vertical profile of the electron distribution at the time of the transmission [2].

Radio pulses travel slower within the ionosphere than those in the free space due to the dispersive nature. Also, due to inhomogeneity of the magnetoplasma, the signals return from a plasma cloud, which is called the turning point. In processing of the signals, the inhomogeneity is typically not taken into account and the signals are assumed to travel with speed of light in vacuum [3], [4]. Therefore, the apparent height of a reflection is called as the virtual height. Moreover, a unique relationship exists between the sounding frequency and the ionization density which reflects it. The process of reconstructing electron density with respect to height and estimating critical parameters of the ionogram with the above assumptions is known as the ionogram scaling or true height analysis [5], [6].

Gokhan Gok is with the Department of Electrical and Electronics Engineering of Bilkent University, Ankara, Turkey (email:ggok@ee.bilkent.edu.tr).

Gokhan Gok and Yasar Kemal Alp are with the Radar, Electronic Warfare and Intelligence Systems Division, ASELSAN AS, Ankara, Turkey.

Orhan Arikan is with the Department of Electrical and Electronics Engineering of Bilkent University, Ankara, Turkey.

Feza Arikan is with the Department of Electrical and Electronics Engineering of Hacettepe University, Ankara, Turkey.
The earliest ionospheric stations were all placed in temperate latitudes for scientific purposes, where the echo traces on the ionograms could be manually scaled by classifying a few patterns which are easily recognized. Ionosonde network has been expanded rapidly during the Second World War as a result of the need to make predictions of radio propagation over the Earth [5]. Rapid growth of measured data forced researchers to use the same detailed conventions and methods. Consequently, different handbooks have been prepared to standardize the manual scaling of the ionograms [5], [6]. In recent years, due to the growing interest in monitoring of the ionosphere for the purposes of improved positioning, navigation and HF communication, the need for near-real time and reliable scalings become more important. As a result, automatic ionogram scaling tools are implemented as signal processing algorithms to extract important ionospheric parameters such as $f_{o} F 2$, the critical frequency, and $h_{m} F 2$, the maximum ionization height, of the F2 layer.

POLynomial ANalysis, POLAN [2], is the first attempt that aims to give consistent results without an operator intervention. The POLAN uses polynomials with any desired degree to model the electron density profile and obtain a weighted least square solution for the polynomial parameters to reconstruct the electron density profile. One of the most widely used automatic scaling tool is the Automatic Real Time Ionogram Scaler with True Height (ARTIST) [7]. ARTIST is deployed as a part of the Digisonde [8] data processing system and it uses N(h) for Personal Computers (NhPC) [9] algorithm to obtain the electron density profile by taking full advantage of the processing power provided by the personal computers of the era. During the scaling process, NhPC expresses the electron density profile of each layer as shifted Chebyshev polynomials and the set of coefficients are calculated to reconstruct the electron density profile [9]. Although ARTIST is considered as the most accomplished algorithm, ionogram scaling is a highly challenging task. As discussed in [10], comparisons reveal that ARTIST is able to provide reliable scalings for about $93 \%$ of the time which indicates that there is a room for improvement. As a result, researchers are trying different techniques for ionogram scaling. Such an attempt is the AUTOSCALA technique given in [11] which adjusts the parameters of a model to find the best electron density profile that minimizes the root mean square error between the measured ionogram and the generated ionogram of the model. In [12], an effort is made to improve the AUTOSCALA by proposing a method for calculating the ionogram for a given electron profile in a fast and robust manner.

Apart from the complete true height analysis, some methods focus on only estimating the critical parameters of the elec- 
(a)

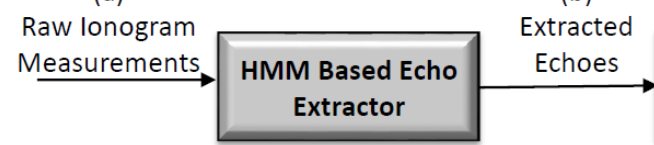

(b)

$\longrightarrow$

(c) Extracted

$\underset{\widehat{f}_{O}}{\mathrm{E} 2} \longrightarrow$

\section{Model Parameter} Optimization Frequency Estimation
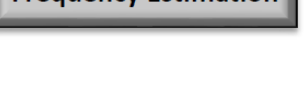

[Date, Time, Location

[Date, Time, Location]

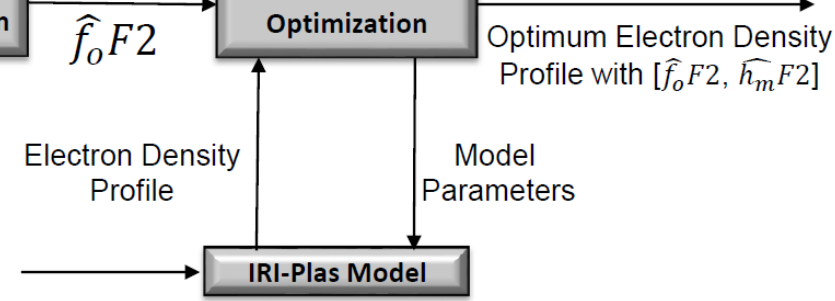

Fig. 1. Flowchart of ISED. (a,b) Raw ionogram measurements and extraction of actual echoes is shown in Fig.3. (c) Estimation of F2 layer critical frequency is shown in Fig.4. (d) Ionogram trace and corresponding electron profile densities calculated by ISED are shown in Fig.6 and Fig.7 respectively.

tron density profile or propose pre/post-processing techniques that could improve the results of existing scaling tools. For example, in [13] and [14], a method for estimating critical frequency parameter $F 2$ layer and $E$ layer are presented respectively. The technique that is proposed in [15] estimates the critical frequency of the F2 layer using the image processing technique proposed in [16]. In [17] and [18], signal processing techniques for clearing artifacts and noise like components that are commonly observed in ionograms are proposed. In [19], an expert system called QualScan is proposed to survey and assign a quality factor to the outputs of the ARTIST in order to highlight the unreliable scalings. In a similar study given in [20], a method for identifying incorrect scalings that are usually observed under disturbed conditions is proposed.

In this study, a novel two stage method for Ionogram Scaling and Electron Density reconstruction (ISED) is proposed. As shown in Fig.1, in the first stage of the ISED, actual echoes in the measurements are identified, where the prior information about the characteristics of the ionospheric reflections and measured amplitudes of echoes are utilized by an Hidden Markov Model (HMM) [21], [22]. Then, the critical frequency of the ionogram, $f_{o} F 2$, is estimated by using the extracted echoes. In the second stage of ISED, by using the Golden Section search method [23], $h_{m} F 2$ parameter is estimated as the solution of the constructed model based nonlinear optimization and the best electron density generated that fits to the recorded ionogram is obtained. Performance of the ISED is demonstrated over a large data set from multiple ionosonde stations. First data set includes ionosonde measurements that are obtained between the dates of 10-Jan-2015 and 02-June2015 at a representative midlatitude ionosonde station located in Pruhonice, Czech Republic with geographic coordinates of $\left[50.00^{\circ} \mathrm{N}, 14.60^{\circ} \mathrm{E}\right]$. Second data set includes ionosonde measurements that are obtained between January 2021 and March 2021, which recorded at a high latitude station located in Eielson, United States with geographic coordinates of $\left[64.66^{\circ} \mathrm{N}, 212.93^{\circ} \mathrm{E}\right]$. Last data set consists of measurements that are obtained in January 2021 of a equatorial station located in Sao Luis, Brazil with geographic coordinates of $\left[-2.6^{\circ} \mathrm{N}\right.$, $\left.315.8^{\circ} \mathrm{E}\right]$.

This paper is organized as follows. In Section II, flow of the proposed ISED algorithm is briefly discussed and the signal model for ionogram output is given. In Section III, the HMM model for ionogram trace extraction is constructed. In Section $\mathrm{IV}$, the technique for the reconstruction of the electron density profile is proposed. In Section V, the performance of ISED is demonstrated.

\section{BRIEF OVERVIEW OF ISED AND SIGNAL MODEL}

Ionograms are graphs of virtual height signal reflections plotted against frequency of the signal. The ionogram can contain echoes from both the near vertical incidence where the transmitter and receiver are co-located and the oblique incidence where transmitter and receiver are located quite far from each other. At this stage, ISED algorithm is designed to process the near vertical incidence echoes. However, it can be adapted for the scaling of oblique ionograms.

The Digisonde DPS4-D [8] is one of the most widely used digital ionosonde that incorporates latest capabilities of RF circuitry and digital signal processing. It converts the round trip delays of the received echoes with a resolution of $2.5 \mathrm{~km}$ at each frequency [8]. Also, amplitudes of the received echoes are measured if there exist a detection in the corresponding range and frequency bin. Operating frequencies of ionosondes are in between $1 \mathrm{MHz}$ and $20 \mathrm{MHz}$, where the frequency resolution is usually set manually by the ionosonde operator.

Considering the ionogram output of the Digisonde DPS4D, ionogram measurement with $K$ distinct frequencies and $N$ distinct virtual heights is modelled as $K \times N$ matrix $\boldsymbol{M}$ such that $M_{k n}=V_{k n}$ where $V_{k n}$ is the measured power at frequency $f_{k}$, height $h_{n}$. For frequency and range bins where there is no detection, amplitudes are set to 0 .

ISED consists of two main signal processing stages. As shown in Fig.1, in the first stage, an HMM, designed specifically for extracting echoes that are received due to reflections from the magnetoplasma up to the maximum ionization height and removing multi-hops and artifacts from the ionogram data, is employed. Hence, echoes that are reflected both from $\mathrm{E}$ and $\mathrm{F}$ layers of the ionosphere are extracted in this step. HMM uses specific states to interpolate frequency gaps in the measurements whereas ARTIST handles the missing frequency points by only using the available trace segments to obtain the electron density profile [7], [8], [24]. In the second stage of the ISED, extracted echoes are used for estimating the critical frequency of $F 2$ layer which plays a crucial role in scaling. Then, a model based optimization problem constructed such 


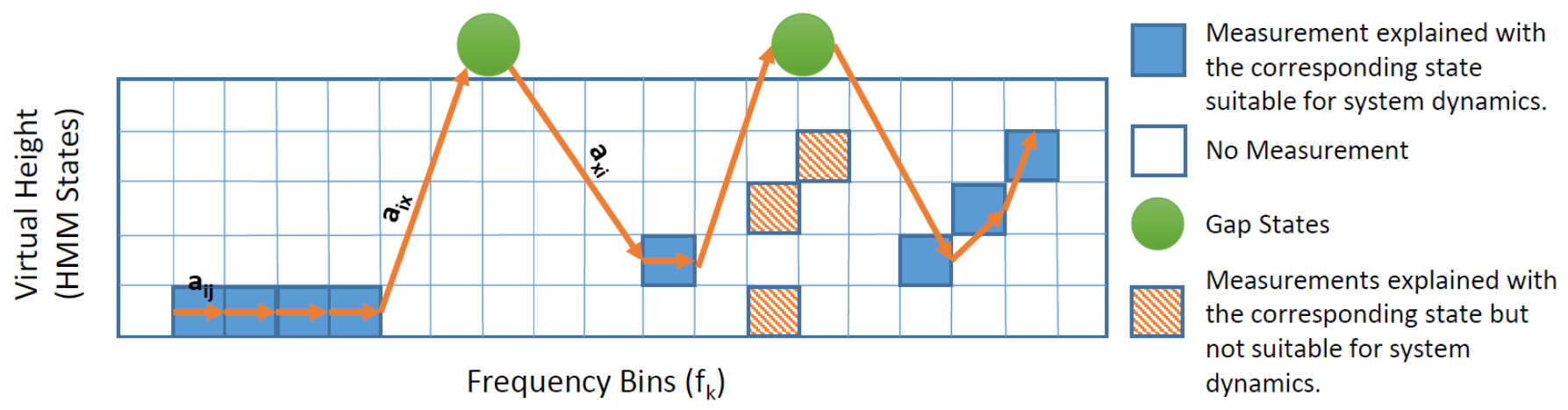

Fig. 2. HMM visualization for echo extraction.

that the electron density that best fits the HMM output is obtained. In the next section, details of the HMM based echo extraction is given.

\section{Hidden Markov Model Based Ionogram Echo EXTRACTION}

Signals can be broadly modeled by using two different approaches. In the first approach, the signal is assumed to belong to a deterministic signal family. In the second approach, they are modeled as a stochastic processes. For stochastic signals, Hidden Markov Models (HMM) are commonly used [22]. Their flexible mathematical structure enables them to be used in a wide range of practical applications from speech recognition [21] to radar remote sensing applications [25].

An HMM is defined by few model parameters called as states, state transition probabilities, observation probabilities and initial state probabilities. Significance of the aforementioned parameters and details related to ionogram echo extraction by using HMM are given in the following subsections.

\section{A. HMM States for Ionogram Echo Extraction}

In order to adapt HMM to ionogram echo extraction process, we consider a system with $N$ distinct states, $\boldsymbol{S}_{\boldsymbol{h}}=$ $\left\{S_{1}, S_{2}, \ldots, S_{N}\right\}$, where each state corresponds to the range bin $h_{n}$ of the ionosonde. We assume that, in each measurement frequency, ionogram trace is in one of these states and by estimating the state sequence using the measurements will provide an accurate ionogram trace. Considering that most ionosonde measurements have frequency gaps as a result of weak reflections or local regulations which restrict transmissions at specific frequencies [19], we additionally define a set of gap states $\boldsymbol{S}_{\boldsymbol{d}}$. By using these gap states, when there is a gap in the observations, model will proceed through the corresponding gap states until it reaches to an appropriate measured echo as illustrated in Fig.2. Therefore, we choose the set of states as $\boldsymbol{S}=\boldsymbol{S}_{\boldsymbol{h}} \cup \boldsymbol{S}_{\boldsymbol{d}}$ for the HMM model.

\section{B. HMM State Transition Probabilities}

As illustrated in Fig.2, it is assumed that the modeled system undergoes a change of state at regular frequency intervals according to the system dynamics which are determined by the set of transition probabilities between states.
Let $f_{k}$ denote the frequency corresponding to state transition and $q_{f_{k}}$ denote the actual state at frequency $f_{k}$. For ionogram echo extraction problem, we consider only the first order Markov Chain where system dynamics are determined by just the current and the predecessor states, i.e,

$$
P\left[q_{f_{k}}=S_{j} \mid q_{f_{k-1}}, \ldots, q_{f_{k-2}}\right]=P\left[q_{f_{k}}=S_{i} \mid q_{f_{k-1}}\right]
$$

Furthermore, we consider only frequency independent state transition probabilities:

$$
a_{i j}=P\left[q_{f_{k}}=S_{j} \mid q_{f_{k-1}}=S_{i}\right], \quad 1 \leq i, j \leq N .
$$

For simplicity, the matrix $\boldsymbol{A}=\left\{a_{i j}\right\}$ will be used to denote the state transition probabilities.

Note that the state transition coefficients must obey the standard stochastic constraints:

$$
\begin{aligned}
a_{i j} & \geq 0, \\
\sum_{j=1}^{N} a_{i j} & =1 .
\end{aligned}
$$

To adapt HMM to ionogram traces, larger transition probabilities are assigned for virtual heights that are close to each other so that the extracted ionogram traces are smoother. Moreover, ionogram traces are typically an increasing function of the frequency hence designed model should be encouraged to jump to a higher virtual height. Considering these properties along with the constraints given in (3), the transition probabilities between states in $S \in \boldsymbol{S}_{\boldsymbol{h}}$ can be set as:

$$
a_{i j}= \begin{cases}\gamma e^{-\left(\left|h_{i}-h_{j}\right|\right) / \beta} & , \text { if } 0 \leq(j-i) \leq 80 \\ 0 & , \text { otherwise. }\end{cases}
$$

Here, $\gamma$ is the normalization factor so that $\sum_{j=1}^{N} a_{i j}=1$ and $\beta$ is the constant controlling how fast the transition probabilities attenuate with height difference between states.

We also need to define the transition probabilities for gap states that are defined for the frequency gaps in the measurement. Let $i$ denote the index of the state in $\boldsymbol{S}_{\boldsymbol{h}}$ and $z$ denote the index of the gap state in $\boldsymbol{S}_{\boldsymbol{d}}$. Then, $a_{i z}$ is chosen as:

$$
a_{i z}= \begin{cases}1 /(2 W+1) & , \text { if }|i-z| \leq W \\ 0 & , \text { otherwise. }\end{cases}
$$

Here, $W$ is a parameter controlling how far the model can jump between the gap states and the actual states. Note that, we define $a_{i z}=a_{z i}$. 


\section{The Observation Probability Distribution}

With the above definitions, if we can observe the set of states at each frequency instant, the corresponding stochastic model is called as an observable Markov model. However, this model is too restrictive to be applicable in many practical problems [21]. To overcome this issue, observations of the model can be defined as probabilistic function of the state.

ISED HMM relates the state observation probabilities with ionogram amplitude measurement in a way that favors the virtual height states which has more powerful echo measurements. In other words, observation probability of a given ionogram measurement at frequency $f_{k}$ in state $j$ is set to be proportional to the echo amplitude for each virtual height, i.e,

$$
b_{j}\left(f_{k}\right)=V_{k j}
$$

Here, we assume that amplitude measurements of the ionosonde, $P_{k j}$, are normalized to satisfy $0 \leq P_{k j} \leq 1$.

For simplicity, matrix $\boldsymbol{B}=\left\{P_{k j}\right\}$ will be used to denote the observation probabilities.

\section{Probability Distribution of Initial State}

Initial states of the HMM correspond to the starting point of the ionogram trace at the minimum frequency which is typically around $1 \mathrm{MHz}$. As there are no previous echoes that the trace can be connected to, probability of the initial states, denoted by $\kappa=\left\{\kappa_{i}\right\}$, determines the likelihood of each virtual height being the first state of the trace.

Our analysis on the raw ionogram data from a midlatitude ionosonde indicates that depending on the time of the day and the day of the year, virtual height of the first echo varies between $[90,450] \mathrm{km}$ range. As a result, initial state probabilities are assumed to be uniformly distributed in $h \in[90,450] \mathrm{km}$ for a midlatitude station.

\section{E. Ionogram Trace Extraction using Viterbi Algorithm}

To identify the most probable sequence of states efficiently, Viterbi algorithm can be used [26], [27]. First, define the highest probability along a single path at frequency $f$ as:

$$
\delta_{f}(i)=\max _{q_{1}, q_{2}, \ldots, q_{f-1}} P\left[q_{1}, q_{2}, \ldots q_{f}=i, O_{1}, O_{2} \ldots O_{f} \mid \ell\right]
$$

where $\ell=(\boldsymbol{A}, \boldsymbol{B}, \boldsymbol{\kappa})$ denotes the set of HMM parameters given in the previous sections and $O_{k}$ denotes the observations at frequency $f_{k}$. By induction, the highest probability at frequency $f+1$ can be written as:

$$
\delta_{f+1}(j)=\max _{i}\left[\delta_{f}(i) a_{i j}\right] b_{j}(f+1) .
$$

To obtain the optimum maximum likelihood (ML) state sequence, argument which maximizes (8) can be tracked and the ML optimum state sequence with probability $P^{*}$ in (13) can be determined using the Viterbi algorithm given in Algorithm 1. Viterbi algorithm obtains the optimal state $q_{f_{K}}^{*}$ at the maximum frequency as given in (14) and backtracks the optimal state sequence using the variable $\Psi_{f}$ given in (12) which is calculated at each frequency. In Fig. 3, the optimum state sequence tracked by the HMM is shown with the black

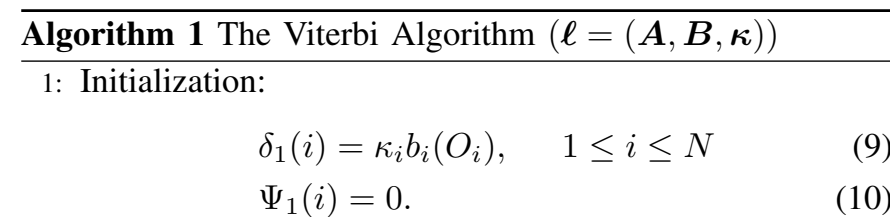

2: Recursion:

$$
\begin{array}{rr}
\delta_{f}(j)=\max _{1 \leq i \leq N}\left[\delta_{f-1}(i) a_{i j}\right] b_{j}\left(O_{f}\right), & f_{2} \leq f \leq f_{K} \\
& 1 \leq j \leq N \\
\Psi_{f}(j)=\underset{1 \leq i \leq N}{\arg \max }\left[\delta_{f-1}(i) a_{i j}\right], & f_{2} \leq f \leq f_{K} \\
1 & \leq j \leq N
\end{array}
$$

3: Termination:

$$
\begin{aligned}
P^{*} & =\max _{1 \leq i \leq N}\left[\delta_{f_{K}}(i)\right], \\
q_{f_{K}}^{*} & =\underset{1 \leq i \leq N}{\arg \max }\left[\delta_{f_{K}}(i)\right] .
\end{aligned}
$$

4: State Sequence backtracking:

$$
q_{f}^{*}=\Psi_{f+1}\left(q_{f+1}^{*}\right), \quad f=f_{K-1}, f_{K-2}, \ldots, f_{1} .
$$

curve. Here, it can be observed that HMM trace successfully tracks the echoes of $\mathrm{F}$ layer.

\section{Electron Density Reconstruction}

\section{A. Estimation of the Critical Frequency for F2 Layer}

Critical frequency parameter of the F2 layer, denoted by $f_{o} F 2$, is the maximum frequency at which an echo is received. Beyond this frequency, electromagnetic waves traverse the ionosphere. Hence, accurate estimation of $f_{o} F 2$ plays a critical part in the reconstruction of the electron density.

Also, tracking quality or the likelihood of the state sequence selected by the HMM also provides valuable information about the $f_{o} F 2$ parameter. As shown with the black curve in Fig.3, HMM tracks the correct echoes until the critical frequency. After the critical frequency, HMM tends to select echoes with higher measured amplitude and follow the echoes that spread both in frequency and height, which decreases the likelihood of the state sequence. Unfortunately, there are other factors which also decrease the likelihood of a given state sequence such as frequency gaps in the measurements or weak echoes in different frequencies. As a result, tracking quality can not be directly employed by using the likelihood of the sequence given in (8). Instead, as a post-processing step, likelihood of the state sequence can be calculated using (8) by assuming that observation probabilities for the chosen states are $\hat{b}_{j}(f)=1$. Then, the coarse estimate of the critical frequency, $\widehat{f}_{o} F 2_{c}$, can be found as:

$$
\widehat{f}_{o} F 2_{c}=\underset{f}{\arg \min }(\delta(f+1) / \delta(f))
$$

In order to estimate $f_{o} F 2$ accurately, the total received power at each frequency can be utilized. However, detections 


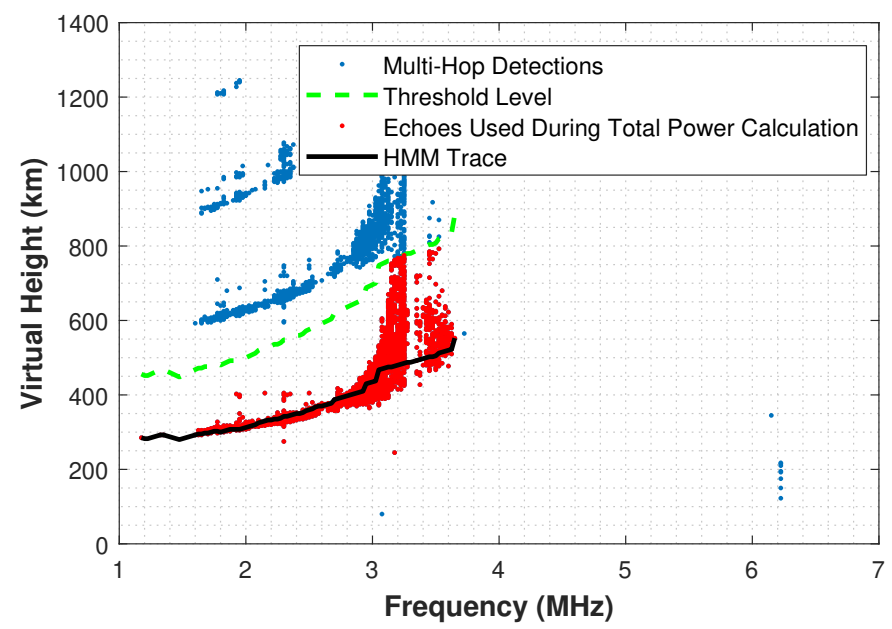

Fig. 3. Calculation of threshold level for eliminating multihop reflections. Red dots show the echoes that are received from the F layer of the ionosphere. Black curve shows optimal state sequence that is tracked by the HMM. Green dashed curve is the threshold level set using the HMM trace. Blue dots are the multi-hop detections eliminated by the HMM trace.(Pruhonice Station, 10-Jan-2015 00:45 UTC)

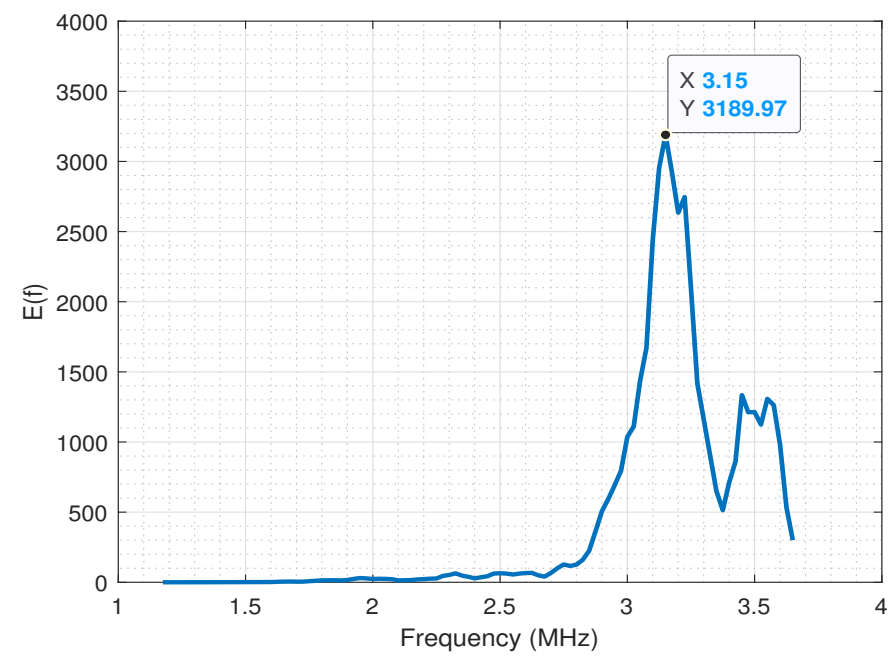

Fig. 4. Total power for each frequency is obtained by integrating the received power for each frequency component. (Pruhonice Station, 10-Jan-2015 00:45 UTC)

due to multihop reflections (multiple reflections from ionosphere and earth surface) also affect the total received power at each frequency. For robust estimation of $f_{o} F 2$, multihop detections that do not contribute to electron density reconstruction has to be removed. This can be achieved by using the ionogram trace generated by the HMM denoted by $h_{H}(f)$. For each frequency $f$, echo amplitudes in $\boldsymbol{M}=\left\{P_{f k}\right\}$ that satisfy the relation $k \geq T$ is set to 0 . Here, $T=\left(\alpha \times h_{H}(f)\right)$ denotes the threshold level and $\alpha<2$ should be satisfied to eliminate multihop reflections as they are observed at twice the height of the actual ionospheric reflection. The threshold level is shown on an example with the green curve in Fig.3 for $\alpha=1.6$.

Following the multihop removing process, total power at each frequency weighted with the coarse estimate is calculated as:

$$
E\left(f_{k}\right)=\sum_{n=1}^{N} V_{k n} e^{-\left(f_{k}-\widehat{f}_{o} F 2_{c}\right)^{2}},
$$

where $N$ denotes the total number of observation heights. An example of calculated $E\left(f_{k}\right)$ which is given in (17) is shown in Fig. 4. Then, $f_{o} F 2$ parameter is estimated as:

$$
\widehat{f}_{o} F 2=\underset{f_{\max }-f_{k} \leq 2 \mathrm{MHz}}{\arg \max } E\left(f_{k}\right)
$$

where $f_{\text {max }}$ is the maximum observed frequency in $\mathrm{MHz}$. By imposing the constraint $\left(f_{\max }-f_{k}\right) \geq 2 \mathrm{MHz}, f_{o} F 2$ parameter estimation becomes robust against spurious measurements that are commonly observed at low frequencies. After this step, $h_{H}(f)$ is modified so that the maximum frequency in the extracted trace is $f_{o} F 2$.

\section{B. Model Based Vertical Electron Density Reconstruction}

In order to complete the automatic scaling process, underlying electron density distribution has to be reconstructed in a way that best fits to the extracted trace by the HMM. To achieve this task, we follow a model based approach. One of the most commonly used model of the ionosphere is the International Reference Ionosphere (IRI) model [28], [29], which has been regularly updated as the new data and techniques available over time [30], [31]. Here, we employ the International Reference Ionosphere extended to Plasmasphere (IRI-Plas) [32], [33] due to the facts that the range of IRI-Plas is extended to the $20,000 \mathrm{~km}$, which is the orbital height of Global Positioning System (GPS) satellites, and also, IRI-Plas has the capability of ingesting Total Electron Content (TEC) as an input for further scaling the electron density as described in [34], [35].

The optimization problem in terms of the IRI-Plas $h_{m} F 2$ parameter is defined as:

$\widehat{h}_{m} F 2=\underset{h_{m} F 2}{\arg \min } \sum_{k \in \boldsymbol{K}}\left(h_{H}\left(f_{k}\right)-h_{I}\left(f_{k}, \widehat{f}_{o} F 2 ; h_{m} F 2\right)\right)^{2}$.

Here, $h_{H}\left(f_{k}\right)$ denotes the trace extracted by HMM and $h_{I}\left(f_{k}, \widehat{f}_{o} F 2 ; h_{m} F 2\right)$ denotes the IRI-Plas trace obtained for the given parameter set $\left\{f_{k}, \widehat{f}_{o} F 2 ; h_{m} F 2\right\}$ at the acquisition time of the data and the geographical location of the ionosonde.

Optimization problem given in (19) is a nonlinear function of $h_{m} F 2$ and it has a convex cost surface. By using the relation between the virtual height and the electron density distribution which is detailed in the Section IV-C, it can be shown that $h_{I}\left(f_{k}, \widehat{f}_{o} F 2, h_{m} F 2+\delta h\right) \approx h_{I}\left(f_{k}, \widehat{f}_{o} F 2, h_{m} F 2\right)+\delta h^{\prime}$. In other words, the IRI-Plas trace is shifted upward or downward as a function of the $h_{m} F 2$ in a nonlinear fashion. This property is demonstrated on a specific example in Fig.5. Using this property, the optimization problem in (19) can be solved by using a nonlinear convex optimization technique such as the Golden Section search [23]. Golden Section search, given in Algorithm 2, can converge to the local minimum of the cost function given in (19). In Fig.7, electron density profile that is obtained using the proposed model based technique is given for the ionogram shown in Fig.6. 


$$
\begin{gathered}
\mu^{2}(f, h)=1-\frac{X(1-X)}{1-X-\frac{1}{2} Y^{2} \sin ^{2}(\theta)+\sqrt{\left(\frac{1}{4}\right) Y^{4} \sin ^{4}(\theta)+(1-X)^{2} Y^{2} \cos ^{2}(\theta)}} \\
\mu^{\prime}(f, h)=\frac{1}{\mu}+\frac{1-X-\mu^{2}+0.5 Y^{2} \cos ^{2}(\theta)\left(1-X^{2}\right)\left(1-\mu^{2}\right)\left(Y^{2} \cos ^{2}(\theta)(1-X)^{2}+0.25 Y^{4} \sin ^{4}(\theta)\right)^{-\frac{1}{2}}}{\mu\left(1-X-\frac{1}{2} Y^{2} \sin ^{2}(\theta)+\sqrt{\left(\frac{1}{4}\right) Y^{4} \sin ^{4}(\theta)+(1-X)^{2} Y^{2} \cos ^{2}(\theta)}\right)}
\end{gathered}
$$

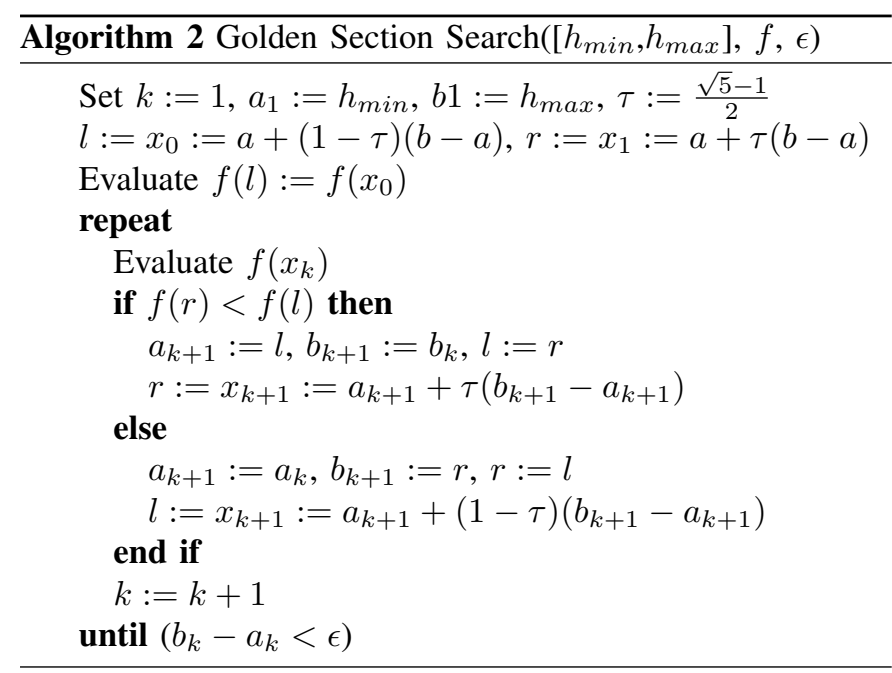

\section{Calculation of Ionogram for IRI-Plas Electron Profile}

Another challenging part of the optimization problem given in (19) is the calculation of the IRI-Plas ionogram, $h_{I}\left(f_{k}, \widehat{f}_{o} F 2 ; h_{m} F 2\right)$, for a given electron density provided by the IRI-Plas model. The half of the round trip delay of an ionosonde pulse can be written as:

$$
\tau(f)=\frac{1}{c} \int_{0}^{h_{r}(f)} \mu^{\prime}(f, h) d h .
$$

Here, $h_{r}(f)$ denotes true reflection height, $c$ is the speed of propagation in free space and $\mu^{\prime}(h, f)$ denotes the group refractive index which can be calculated by

$$
\mu^{\prime}=\mu+f \frac{\partial \mu}{\partial f}
$$

where $\mu$ is the refractive index given by the Appleton-Hartree equation [3] that is given in (20) for the ordinary wave where the effect of electron collisions are neglected. In (20), $w_{p}=$ $\sqrt{\left(N_{e}(h) e^{2}\right) /\left(\epsilon_{0} m_{e}\right)}$ is the plasma frequency; $X=w_{p}^{2} / w^{2}$, $w_{H}=B_{0}|e| / m_{e}$ is the gyro frequency; $B_{0}$ is the magnitude of the magnetic flux density; $Y=w_{H} / w, N_{e}(h)$ is the vertical electron density profile; $\epsilon_{0}$ is the permittivity of free space; $\theta$ is the angle between the earth magnetic field vector and the wave vector; $m_{e}$ is the mass of the electron, and $e$ is the charge of the electron. Substituting the refractive index given in (20) into (23), group refractive index can be obtained as (21) [3].

At the true reflection height, electron density reaches the critical value, i.e, $\mu\left(h_{r}(f), f\right)=0$, beyond which there is no reflection at the given frequency. For the ordinary ray case, reflection condition $\mu(h, f)=0$ can be simplified as:

$$
X(h)=\frac{N_{e}(h) e^{2}}{\epsilon_{0} m_{e}(2 \pi f)^{2}}=1 .
$$

It is more convenient to express the ionosonde data in terms of the virtual height $h^{\prime}(f)=\tau(f) c$ which is the distance that ionosonde signal would have traveled in free space for a given delay time $\tau(f)$. Using this relation along with Eq.(22), the virtual height can be calculated as:

$$
h^{\prime}(f)=\int_{0}^{h_{r}(f)} \mu^{\prime}(f, h) d h .
$$

The integral given in (25) forms the foundation of the relationship between the ionosonde echoes and the vertical electron density profile. Therefore, for a given electron density profile $N_{e}(h)$, resulting ionogram trace can be found by numerically computing (25). Since the integrand tends to infinity at the upper boundary, simple Riemann Sum approximation does not provide reliable results [12], [36]. Here, adaptive Gauss-Kronrod quadrature method [37] where the integrated function $f(x)$ is expressed as $f(x)=w(x) g(x)$ in order to regularize the problem and overcome the difficulties caused by the singularities at the boundaries.

Another important aspect of the relation between the electron density profile and the virtual height is that, shifting the electron density profile by changing the maximum ionization height parameter $h_{m} F 2$ in one direction results in shifting the virtual height profile in the same direction. This property can be easily observed by rewriting the integral equation given in (25) for a shifted electron density profile. Hence, optimization problem given in (19) that is introduced in Sec. IV-B can be solved using the Golden Section search technique.

\section{Results Obtained on Ionosonde Data}

The proposed ISED algorithm for estimation of critical frequency and reconstruction of electron density profile is applied to raw ionogram outputs of three different ionosonde stations with different latitudes to demonstrate the performance of ISED in global scale. The ionosondes produce raw ionograms and these ionograms are automatically scaled by using ARTIST (version 5002). Typically, the temporal resolution of the ionosonde data is 15 minutes and the frequency resolution of the data is $0.025 \mathrm{MHz}$. The ISED algorithm is applied to raw ionograms and the F2 layer parameters, namely, $f_{o} F 2$ and $h_{m} F 2$, and also the vertical electron density profile are estimated as discussed in the previous sections. For each application, electron density profile of the IRI-Plas algorithm is 


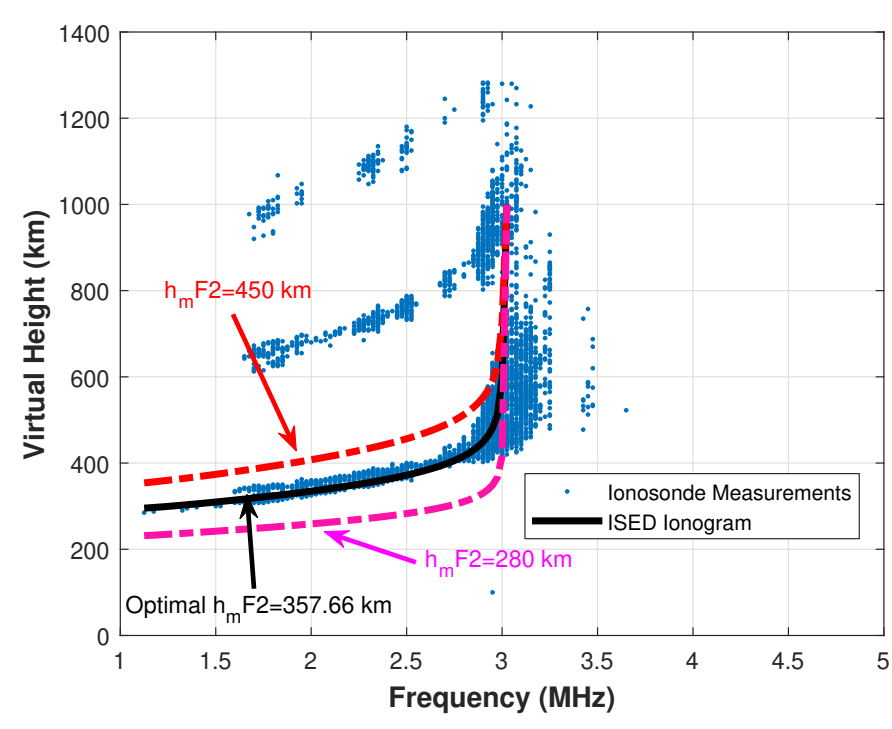

Fig. 5. Ionogram trace calculated for the optimal IRI-Plas solution that best fits the HMM trace. (Pruhonice Station, 10-Jan-2015 01:15 UTC)

obtained for the given date and time of the measurement at the coordinates of the corresponding station. The model outputs without any optimization and the corresponding computed ionograms, which are calculated using (25), are also included in the following figures to demonstrate the importance of the optimization step carried out by the ISED.

First data set includes measurements of a midlatitude station, Pruhonice in Czech Republic, for the time period between January 10, 2015 and June 2, 2015. The ionosonde at Pruhonice (PQ052) is Digital Portable Sounder (DPS-4D) located at geographic coordinates $\left[50.00^{\circ} \mathrm{N}, 14.60^{\circ} \mathrm{E}\right]$; geomagnetic coordinates $\left[49.34^{\circ} \mathrm{N}, 98.57^{\circ} \mathrm{E}\right]$. The ISED algorithm is applied to 13,812 ionograms and the algorithm outputs are compared with those of the ARTIST and manually scaling results considering the entire profile to obtain the performance of each automatic scaling techniques. In 12,321 ionograms out of a total of 13,812 ionograms ( $89.21 \%$ of the total) the ISED and the ARTIST produced very similar outputs which are also consistent with manually scaled ionograms. An example of such an ionogram is provided in Fig.6 where the F-layer returns (given in blue dots on raw ionogram) are spread in a wide range of frequencies on January 12, 2015 at 05:30 UTC. Here, both the ARTIST (red dash dot curve) and the ISED (solid black curve) traces follow similar tracks and resolve spread-F ionogram. Similary, in Fig.8, both ARTIST and ISED successfully track E and F layer echoes and obtain similar electron density profiles as shown in Fig.9 until the F2 layer peak density is reached. Here, it should be noted that ISED processing does not estimate E layer frequency. Instead, IRIPlas model determines the electron density profile and the critical frequency of the $\mathrm{E}$ layer depending on the model inputs.

In 1,230 ionograms out of a total of 13,812 ionograms ( $8.90 \%$ of the total), ISED is able to improve the results of the ARTIST. Such an example is shown in Fig.10. Similar examples can be easily identified in Fig.11 and Fig.12 where the estimated parameters of both ARTIST and the ISED are shown for the first 1,000 ionograms of the dataset. As it can be seen in Fig.12, ARTIST algorithm fails to track correct ionogram traces and estimates $f_{o} F 2$ parameter lower than it should be which also affects the estimated $h_{m} F 2$ parameter as shown in Fig.11. During the analysis, these cases could not be linked to any particular event in the ionosphere and the ARTIST ionogram is quite similar to the one that is shown in Fig.10. On the other hand, ISED is able to track the ionospheric echoes in a robust manner using the HMM.

2014 was the solar maximum year of $24^{\text {th }}$ solar cycle so solar activity was slowing down in 2015. During the period from January 10 to June 2, there were about 10 geomagnetically disturbed periods including the severe St. Patrick's Day storm that extended from March 17 to March 22, 2015. In such cases, ionograms might become too complicated to scale for automatic scaling techniques. Such an example is shown in Fig.13 for a sporadic E ionogram example where both ARTIST and ISED fails. In 261 out of a total of 13,812, which corresponds to $1.88 \%$, ionograms there are problems with the preprocessing of the ionograms, and thus, the automatic scaling can not be performed. To summarize our results for Pruhonice station, Fig.14 shows the monthly performance comparison between ARTIST and ISED for the time period from January 2015 to May 2015. Results indicate that, ISED is able output improved scalings compared to ARTIST for each month of the data set obtained in Pruhonice station.

Second data set consists of measurements belonging to a high latitude ionosonde station, Eielson (EI764) located in United States with geographic coordinates of $\left[64.66^{\circ} \mathrm{N}\right.$, $212.93^{\circ} \mathrm{E}$. ISED algorithm is applied to 500 randomly selected ionograms that are obtained during the time period from January 2021 to March 2021. In 478 out of a total of 500 ionograms $(95.6 \%$ of the total), ISED is able to successfully scale the ionograms. As shown on an example in Fig.15, ISED has managed to obtain better ionograms compared to the ARTIST in 46 out of a total of 500 ionograms (9.2\% of the total). Last data set belongs to a equatorial

TABLE I

SUCCESSFUL SCALING RATE OF ARTIST AND ISED FOR MIDLATITUDE, POLAR AND EQUATORIAL STATIONS.

\begin{tabular}{||c|c|c|c||}
\hline Ionosonde Station & Region & $\begin{array}{c}\text { ARTIST Successful } \\
\text { Scaling Rate }\end{array}$ & $\begin{array}{c}\text { ISED Successful } \\
\text { Scaling Rate }\end{array}$ \\
\hline Pruhonice (PQ052) & Mid Latitude & $89.21 \%$ & $98.12 \%$ \\
\hline Eielson (EI764) & High Latitude & $86.4 \%$ & $95.6 \%$ \\
\hline Sao Luis (SAA0K) & Equator & $83.2 \%$ & $86.0 \%$ \\
\hline
\end{tabular}

station Sao Luis (SAA0K) located in Brazil with geographic coordinates of $\left[-2.6^{\circ} \mathrm{N}, 315.8^{\circ} \mathrm{E}\right]$. Experiments for the Sao Luis station is conducted for 500 randomly selected ionograms that is obtained in January 2021. Equatorial ionograms are important topics of investigation as they include large scale F-region irregularities and second hop of sporadic E layer might overlap with F layer echoes [38]. Such an example is given in Fig.16 where there both the ARTIST and the ISED is able to scale the ionogram successfully. Note that, in Fig.16, the ARTIST scaling result follows the measurements slightly better compared to scaling result of the ISED which is due 


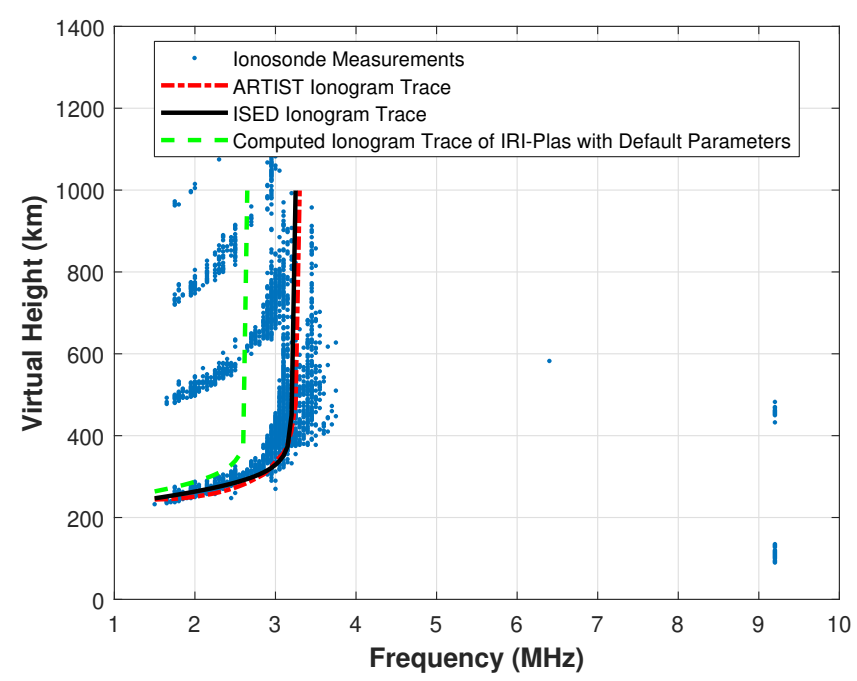

Fig. 6. Ionosonde measurements and comparison of ionogram traces for Pruhonice Station, 12-Jan-2015 05:30 UTC.

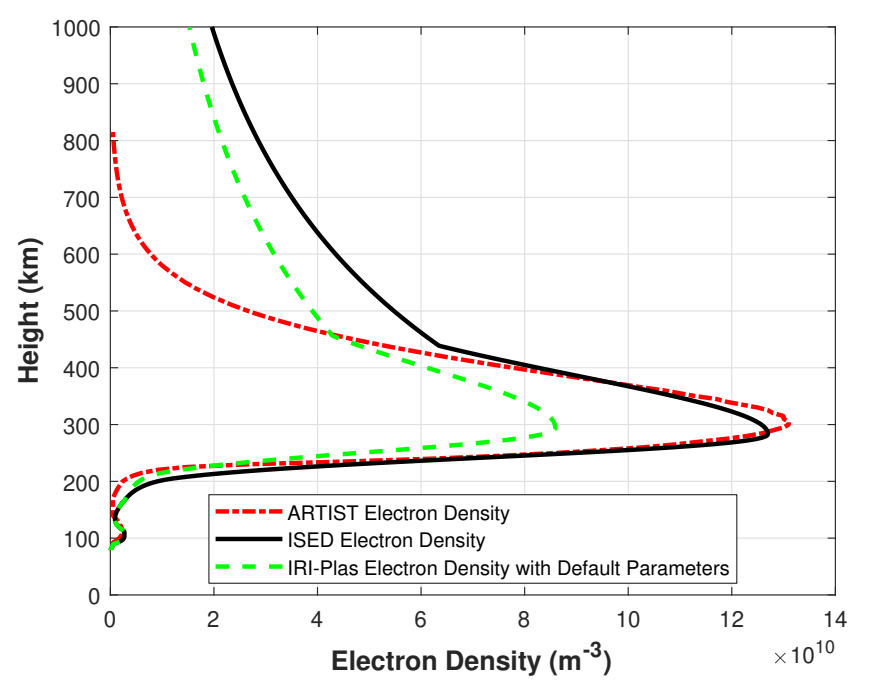

Fig. 7. Comparison of reconstructed electron density profiles for Pruhonice Station, 12-Jan-2015 05:30 UTC.

to the negligible modeling errors introduced by the IRI-Plas model. As summarized in Table I, overall results over a total of 500 ionograms shows that ISED is able to improve the scaling performance by $2.8 \%$ compared to the ARTIST.

\section{CONCLUSION}

In this study, a novel algorithm, ISED, is proposed for ionogram scaling and electron density reconstruction. ISED reconstructions are obtained in two stages. First, by using a Hidden Markov Model, the actual echoes in the ionosonde measurements are identified even if there are significant gaps in the data. Then, electron density distribution profile is reconstructed by solving a model based optimization problem where the IRI-Plas trace with a set of estimated parameters at the data acquisition time and the geographical location of the ionosonde is used as the model. The performance of the ISED is evaluated using 14,812 ionograms recorded at three different ionosonde stations, namely, a midlatitude station, Pruhonice,

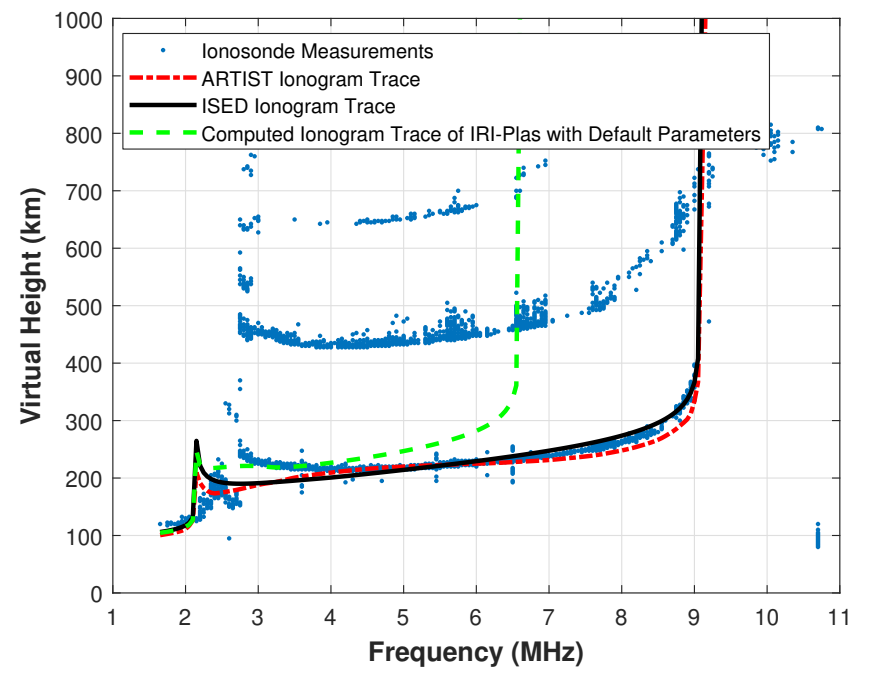

Fig. 8. Ionosonde measurements and comparison of ionogram traces for Pruhonice Station, 11-Jan-2015 08:15 UTC.

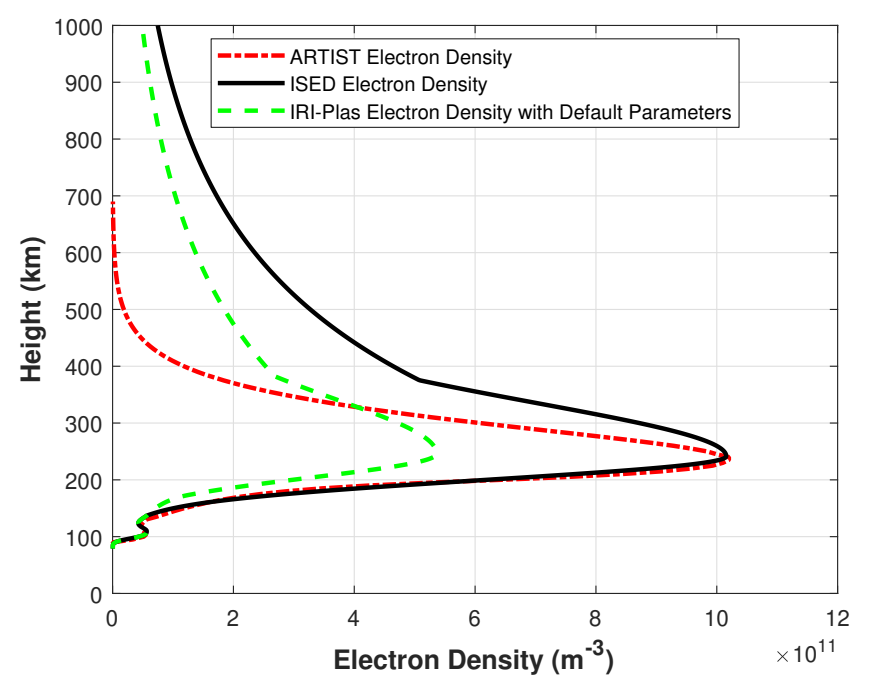

Fig. 9. Comparison of reconstructed electron density profiles for Pruhonice Station, 11-Jan-2015 08:15 UTC.

a high latitude station, Eilson, and an equatorial station, Sao Luis. In $97.6 \%$ of the available ionograms, it is observed that ISED provides accurate electron density reconstructions, which is an improvement about $8.7 \%$ over ARTIST, which is the state of the art ionogram scaling technique. In a future study, we are planning to compare the results ISED for different conditions such as the time of day or quiet and disturbed days. Also, executable version of the ISED will be developed to make it available for public use.

\section{ACKNOWLEDGMENT}

Authors would like to thank Dr. Tamara Gulyaeva from Puskov Institute of Terrestrial Magnetism, Ionosphere and Radio Wave Propagation of Russian Academy of Sciences (IZMIRAN), Russia for her valuable comments. Also, authors would like to thank Dr. Zbyšek Mošna from Institute of Atmospheric Physics, The Czech Academy of Sciences, Czech Republic, for valuable discussions and providing the 


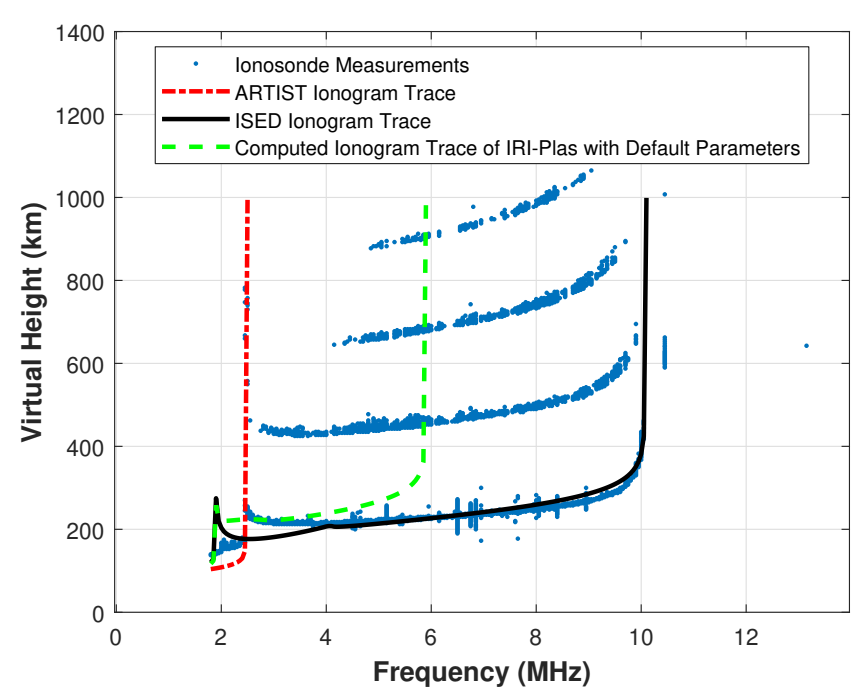

Fig. 10. Ionosonde measurements and comparison of ionogram traces for Pruhonice Station, 12-Jan-2015 07:45 UTC.

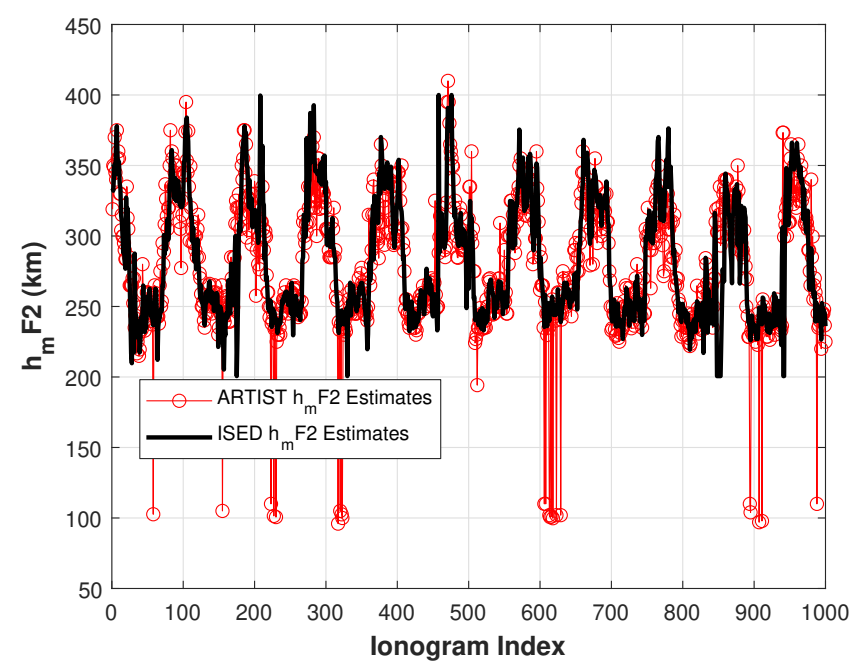

Fig. 11. Estimated $h_{m} F 2$ parameters of ARTIST and ISED for the Pruhonice Station.

data of Pruhonice ionosonde station. Authors wish to express their thanks to anonymous reviewers whose comments and suggestions helped to improve the manuscript. This study is supported by the grant TUBITAK EEEAG 115E915.

\section{REFERENCES}

[1] R. E. Collin, Antennas and radiowave propagation. McGraw-Hill, 1985.

[2] J. Titheridge, "Ionogram analysis with the generalised program polan," 1985.

[3] K. G. Budden, The propagation of radio waves: The theory of radio waves of low power in the ionosphere and magnetosphere. Cambridge University Press, 1988.

[4] K. G. Budden, Radio Waves in the Ionosphere, 2009.

[5] W. R. Piggott and K. Rawer, "Ursi handbook of ionogram interpretation and reduction," 1972

[6] N. Wakai, H. Ohyama, and T. Koizumi, "Manual of ionogram scaling," Radio Research Laboratory, Ministry of Posts and Telecommunications, Japan, 1987.

[7] B. W. Reinisch, R. R. Gamache, J. S. Tang, and D. F. Kitrosser, "Automatic real time ionogram scaler with true height analysis-artist," DTIC Document, Tech. Rep., 1983.

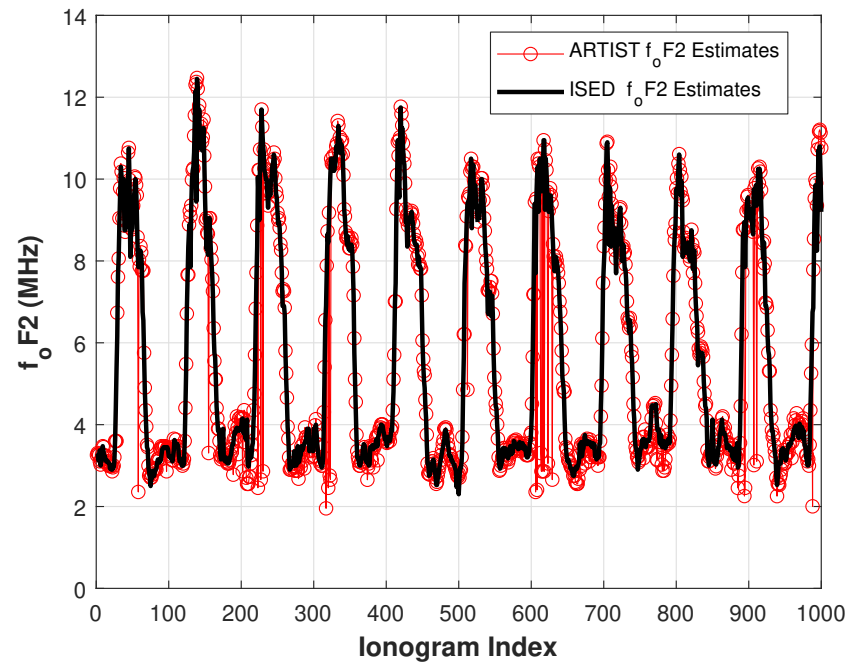

Fig. 12. Estimated $f_{o} F 2$ parameters of ARTIST and ISED for Pruhonice Station.

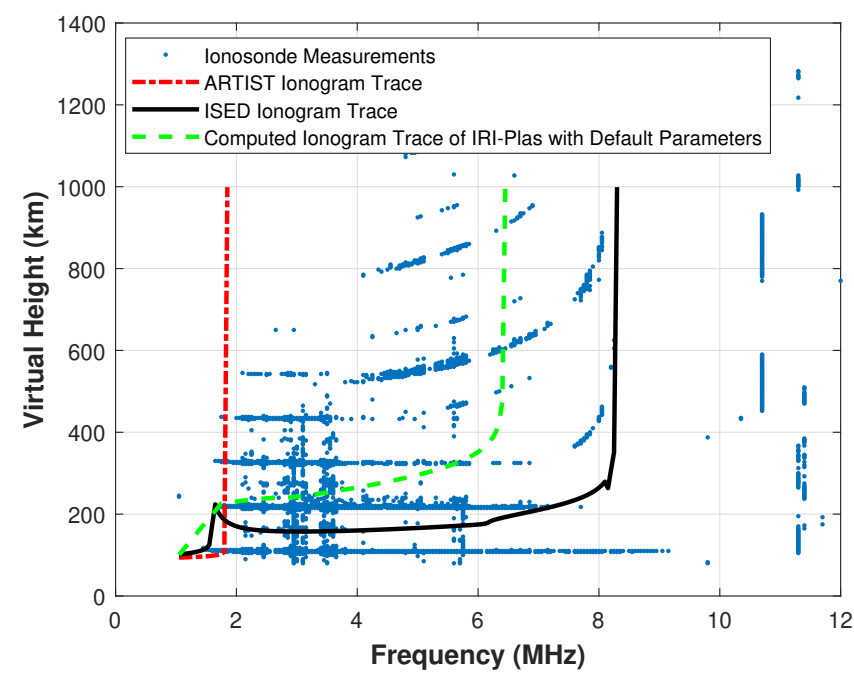

Fig. 13. Ionosonde measurements and comparison of ionogram traces for Pruhonice Station, 30-May-2015 18:30 UTC.

[8] B. W. Reinisch, I. A. Galkin, G. Khmyrov, A. Kozlov, K. Bibl, I. Lisysyan, G. Cheney, X. Huang, D. Kitrosser, V. Paznukhov et al., "New digisonde for research and monitoring applications," Radio Science, vol. 44, no. 01, pp. 1-15, 2009.

[9] X. Huang and B. Reinisch, "Vertical electron density profiles from the digisonde network," Advances in Space Research, vol. 18, no. 6, pp. 121-129, 1996

[10] J. D. Gilbert and R. W. Smith, "A comparison between the automatic ionogram scaling system artist and the standard manual method," Radio Science, vol. 23, no. 06, pp. 968-974, 1988.

[11] C. Scotto, "Electron density profile calculation technique for autoscala ionogram analysis," Advances in Space Research, vol. 44, no. 6, pp. 756-766, 2009.

[12] C. Scotto, M. Pezzopane, and B. Zolesi, "Estimating the vertical electron density profile from an ionogram: On the passage from true to virtual heights via the target function method," Radio Science, vol. 47, no. 1, 2012.

[13] C. Scotto and M. Pezzopane, "A software for automatic scaling of fof 2 and muf (3000) f2 from ionograms," URSI XXVIIth General Assembly, 2002.

[14] C. Scotto and M. Pezzopane, "A method for automatic scaling of sporadic e layers from ionograms," Radio Science, vol. 42, no. 02, pp. $1-5,2007$.

[15] M. Fagre, J. A. Prados, J. Scandaliaris, B. S. Zossi, M. A. Cabrera, 


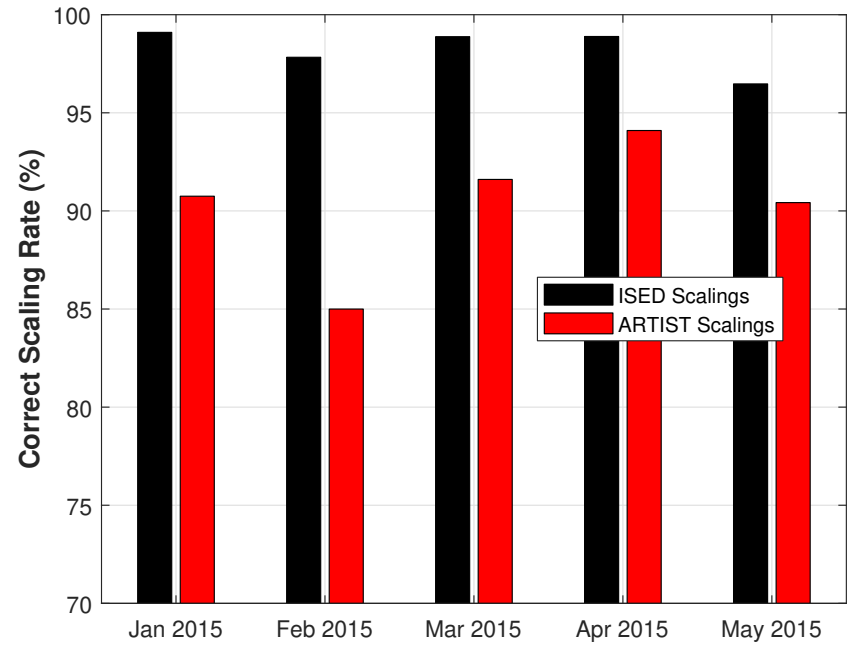

Fig. 14. Monthly performance comparison between January 2015 and May 2015 for the Pruhonice station.

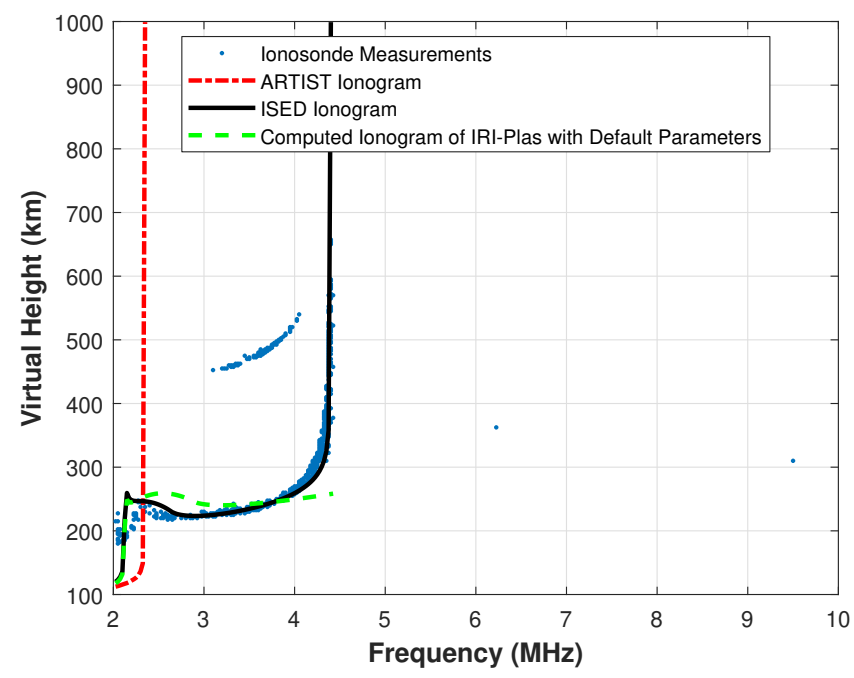

Fig. 15. Ionosonde measurements and comparison of ionogram traces for Eielson Station, 05-Feb-2021 22:30 UTC.

R. G. Ezquer, and A. G. Elias, "Algorithm for automatic scaling of the f-layer using image processing of ionograms," IEEE Transactions on Geoscience and Remote Sensing, 2020.

[16] C. Steger, "An unbiased detector of curvilinear structures," IEEE Transactions on pattern analysis and machine intelligence, vol. 20, no. 2, pp. 113-125, 1998.

[17] F. Arikan, S. Salous, and O. Arikan, "Algorithm for high quality ionograms," Electronics Letters, vol. 36, no. 11, pp. 985-987, 2000.

[18] F. Arikan, O. Arikan, and S. Salous, "A new algorithm for high-quality ionogram generation and analysis," Radio science, vol. 37, no. 1, pp. $1-11,2002$.

[19] L. F. McNamara, "Quality figures and error bars for autoscaled digisonde vertical incidence ionograms," Radio science, vol. 41, no. 04, pp. 1-16, 2006.

[20] L. Rejfek, Z. Mošna, D. Kouba, J. Boška, and D. Burešová, "Application of digital filters to check quality of the automatically scaled ionograms," Journal of Electrical Engineering, vol. 66, no. 3, pp. 164-168, 2015.

[21] L. R. Rabiner, "A tutorial on hidden markov models and selected applications in speech recognition," Proceedings of the IEEE, vol. 77, no. 2, pp. 257-286, 1989.

[22] L. Rabiner and B. Juang, "An introduction to hidden markov models," ieee assp magazine, vol. 3, no. 1, pp. 4-16, 1986.

[23] D. P. Bertsekas, Nonlinear programming. Athena scientific Belmont, 1999.

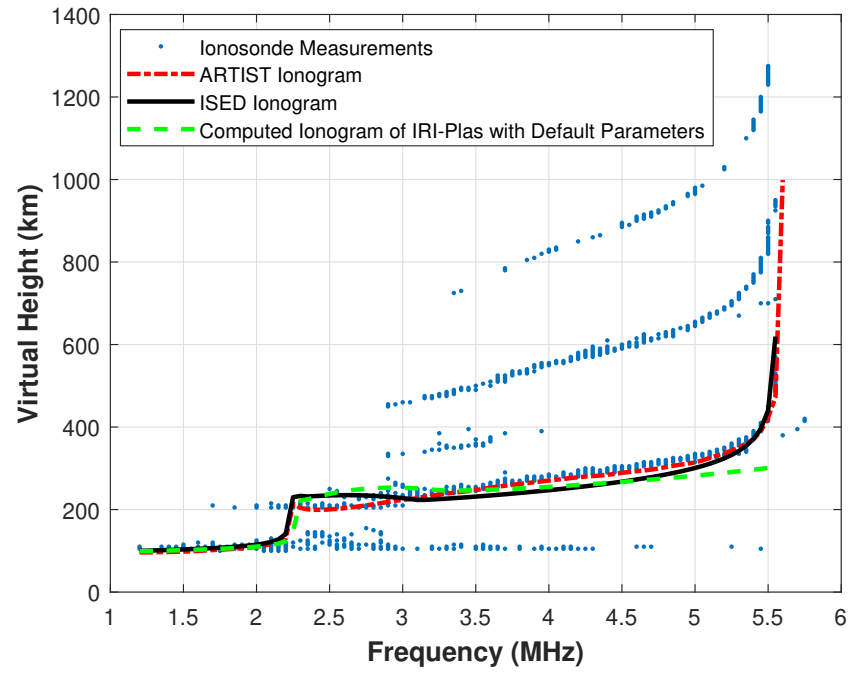

Fig. 16. Ionosonde measurements and comparison of ionogram traces for Sao Luis Station, 01-Jan-2021 09:50 UTC.

[24] B. Reinisch, X. Huang, I. Galkin, V. Paznukhov, and A. Kozlov, "Recent advances in real-time analysis of ionograms and ionospheric drift measurements with digisondes," Journal of Atmospheric and SolarTerrestrial Physics, vol. 67, no. 12, pp. 1054-1062, 2005.

[25] Y. K. Alp, U. Yavuz, and A. Bayri, "Hmm based track-before-detect strategy for fmcw/cw emitter detection," in Signal Processing and Communications Applications Conference (SIU), 2013 21st. IEEE, 2013, pp. 1-4.

[26] A. Viterbi, "Error bounds for convolutional codes and an asymptotically optimum decoding algorithm," IEEE transactions on Information Theory, vol. 13, no. 2, pp. 260-269, 1967.

[27] G. D. Forney, "The viterbi algorithm," Proceedings of the IEEE, vol. 61, no. 3, pp. 268-278, 1973.

[28] K. Rawer, S. Ramakrishnan, and D. Bilitza, "International reference ionosphere, international union of radio science, ursi special report," 1978.

[29] D. Bilitza, "International reference ionosphere, nssdc 90-22," World Data Center A, Rockets and Satellites, Greenbelt, USA, 1990.

[30] D. Bilitza and B. W. Reinisch, "International reference ionosphere 2007: improvements and new parameters," Advances in space research, vol. 42, no. 4, pp. 599-609, 2008.

[31] D. Bilitza, D. Altadill, Y. Zhang, C. Mertens, V. Truhlik, P. Richards, L.A. McKinnell, and B. Reinisch, "The international reference ionosphere 2012-a model of international collaboration," Journal of Space Weather and Space Climate, vol. 4, p. A07, 2014.

[32] T. L. Gulyaeva, X. Huang, and B. W. Reinisch, "Plasmaspheric extension of topside electron density profiles," Advances in Space Research, vol. 29, no. 6, pp. 825-831, 2002.

[33] T. Gulyaeva and D. Bilitza, "Towards iso standard earth ionosphere and plasmasphere model," in New developments in the standard model, 2012, pp. $1-40$.

[34] U. Sezen, T. Gulyaeva, and F. Arikan, "Online computation of international reference ionosphere extended to plasmasphere (iri-plas) model for space weather," Geodesy and Geodynamics, vol. 9, no. 5, pp. 347357, 2018.

[35] U. Sezen, T. L. Gulyaeva, and F. Arikan, "Performance of solar proxy options of iri-plas model for equinox seasons," Journal of Geophysical Research: Space Physics, vol. 123, no. 2, pp. 1441-1456, 2018.

[36] G. Monegato and L. Scuderi, "Numerical integration of functions with boundary singularities," Journal of Computational and Applied Mathematics, vol. 112, no. 1, pp. 201-214, 1999.

[37] L. F. Shampine, "Vectorized adaptive quadrature in matlab," Journal of Computational and Applied Mathematics, vol. 211, no. 2, pp. 131-140, 2008.

[38] V. G. Pillat, P. R. Fagundes, and L. N. F. Guimarães, "Automatically identification of equatorial spread-f occurrence on ionograms," Journal of Atmospheric and Solar-Terrestrial Physics, vol. 135, pp. 118-125, 2015 . 